\title{
Preventive Effects of the Intestine Function Recovery Decoction, a Traditional Chinese Medicine, on Postoperative Intra-Abdominal Adhesion Formation in a Rat Model
}

\author{
Cancan Zhou, ${ }^{1}$ Pengbo Jia, ${ }^{2}$ Zhengdong Jiang, ${ }^{1}$ Ke Chen, ${ }^{1}$ Guanghui Wang, ${ }^{3}$ \\ Kang Wang, ${ }^{3}$ Guangbing Wei, ${ }^{3}$ and $\mathrm{Xuqi} \mathrm{Li}^{3}$ \\ ${ }^{1}$ Department of Hepatobiliary Surgery, The First Affiliated Hospital of Xian Jiaotong University, Xi'an, Shaanxi 710061, China \\ ${ }^{2}$ Department of General Surgery, The First People's Hospital of Xianyang City, Xianyang, Shaanxi 712000, China \\ ${ }^{3}$ Department of General Surgery, The First Affiliated Hospital of Xian Jiaotong University, Xian, Shaanxi 710061, China
}

Correspondence should be addressed to Xuqi Li; lixuqi@163.com

Received 5 August 2016; Revised 18 November 2016; Accepted 5 December 2016

Academic Editor: Ipek Suntar

Copyright (C) 2016 Cancan Zhou et al. This is an open access article distributed under the Creative Commons Attribution License, which permits unrestricted use, distribution, and reproduction in any medium, provided the original work is properly cited.

\begin{abstract}
The intestine function recovery decoction (IFRD) is a traditional Chinese medicine that has been used for the treatment of adhesive intestinal obstruction. In this study, the preventative effects and probable mechanism of the IFRD were investigated in a rat model. We randomly assigned rats to five groups: normal, model, control, low dose IFRD, and high dose IFRD. In the animal model, the caecum wall and parietal peritoneum were abraded to induce intra-abdominal adhesion formation. Seven days after surgery, adhesion scores were assessed using a visual scoring system, and histopathological samples were examined. The levels of serum interleukin-6 (IL-6) and transforming growth factor beta-1 (TGF- $\beta 1$ ) were analysed by an enzyme-linked immunosorbent assay (ELISA). The results showed that a high dose of IFRD reduced the grade of intra-abdominal adhesion in rats. Furthermore, the grades of inflammation, fibrosis, and neovascularization in the high dose IFRD group were significantly lower than those in the control group. The results indicate that the IFRD can prevent intra-abdominal adhesion formation in a rat model. These data suggest that the IFRD may be an effective antiadhesion agent.
\end{abstract}

\section{Introduction}

Intra-abdominal adhesions are a common complication that occurs in $90-95 \%$ of patients who undergo abdominal surgery $[1,2]$. Intra-abdominal adhesion can cause abdominal and pelvic pain, adhesive intestinal obstruction, infertility, and other severe complications [3]. Approximately 10\% of bowel obstructions caused by adhesions require surgery to release the adhesions, with resulting mortality rates of 5$20 \%$ [4]. Operative adhesiolysis results in increased surgical difficulty, prolonged operative duration, and increased risk of bleeding. The situation is even more complicated because approximately $30 \%$ of patients require reoperation for adhesion recurrence [5].

The recurrence rate of adhesive bowel obstruction after treatment using a surgical method is high. Without effective precautions, the recurrence rate is $12 \%$ within 41 months of the initial surgery. The risk of relapse is present even after 20 years [6]. Adhesive bowel obstruction causes endless pain in patients and places a considerable burden on already overtaxed healthcare systems [7]. To date, there is no effective method for preventing intra-abdominal adhesion [8]. Therefore, finding an effective agent or strategy to prevent intraabdominal adhesion is critical [9].

In China and other parts of East Asia, traditional Chinese medicine (TCM) has been used to treat disease for thousands of years. TCM usually works by mixing different types of herbs, which are called formulas or "Fufang." As a complementary treatment, TCM may offer an option for prevention of intra-abdominal adhesion formation. According to TCM theory, intestine function recovery decoction (IFRD) has been used clinically with substantial benefits in treating adhesive intestinal obstruction. However, there is no in vivo experimental evidence showing this effect or likely 
TABLE 1: Constituents of intestine function recovery decoction.

\begin{tabular}{|c|c|c|c|c|}
\hline Chinese name & Latin name & Family & Plant part & Dry weight (g) \\
\hline Dangshen & Codonopsis pilosula Franch. & Campanulaceae & Radix & 15 \\
\hline Baizhu & Atractylodes macrocephala Koidz. & Asteraceae & Rhizoma & 15 \\
\hline Taoren & Prunus persica Batsch & Rosaceae & Semen & 10 \\
\hline Chishao & Paeonia lactiflora Pall. & Ranunculaceae & Radix & 15 \\
\hline Zhiqiao & Citrus aurantium $\mathrm{L}$. & Rutaceae & Fructus & 12 \\
\hline Houpu & Magnolia officinalis Rehd. et Wils. & Magnoliaceae & Cortex & 15 \\
\hline Muxiang & Aucklandia lappa Decne. & Asteraceae & Radix & 15 \\
\hline Huomaren & Cannabis sativa $\mathrm{L}$. & Moraceae & Fructus & 30 \\
\hline Daihuang & Rheum palmatum L. & Polygonaceae & Radix and Rhizoma & 20 \\
\hline Total & & & & 147 \\
\hline
\end{tabular}

pharmacological mechanisms of adhesion prevention. In this study, we demonstrate the effects of the IFRD and explain a likely mechanism for intra-abdominal adhesion prevention in a rat model.

\section{Materials and Methods}

2.1. Preparation of the IFRD. The constituents of the IFRD are shown in Table 1. All of the TCM herbs were purchased from the pharmacy of the First Affiliated Hospital of Xi'an Jiaotong University. Each herb was identified and authenticated by the head of the department and herbal medicinal botanist. Per the Pharmacopoeia of China (version 2010), exact amounts of component herbs were weighed according to the classic percentages and mixed well. The mixture was soaked in distilled water for $30 \mathrm{~min}$ and then boiled in eight volumes of water $(\mathrm{v} / \mathrm{w})$ for $1 \mathrm{~h}$ in herb-extracting machine. This preparation followed the ancient method and was also identical to the clinical preparation. The supernatant was orally administered to the rats in the experiments. The concentration of the IFRD is expressed as the total dry weight of the crude herbs per millilitre in decoction.

2.2. Operation and Postoperative Intervention. SpragueDawley rats weighing 200-250 g were purchased from the Experimental Animal Centre of Xian Jiaotong University (SYXK2012-003). All the rats were fed at room temperature $\left(22 \pm 2^{\circ} \mathrm{C}\right)$. They were allowed to drink freely and were provided with standard rat chow. All the procedures were authorized by the Animal Ethics Committee of Xi'an Jiaotong University (XJTULAC2016-410). The animals were randomly divided into five groups: normal, model, control, low dose, and high dose IFRD groups. All the rats were anesthetized using methoxyflurane prior to surgery. The skin was shaved and sterilized using iodine solution. As previously described [10], a 2 to $3 \mathrm{~cm}$ incision was made. The caecum wall and corresponding parietal peritoneum were scrubbed with sterile gauze until punctate haemorrhage occurred. The area of the injured wall of the caecum was approximately $2-3 \mathrm{~cm}^{2}$. After exposure to air for $5 \mathrm{~min}$, the bowel loop was arranged and returned to its original position. In the normal group, the caecum wall and corresponding parietal peritoneum were not scrubbed.
TABLE 2: Steps of operation and postoperative intervention for each group.

\begin{tabular}{|c|c|c|c|}
\hline Groups & $\begin{array}{l}\text { Abdominal } \\
\text { incision }\end{array}$ & $\begin{array}{l}\text { Peritoneum } \\
\text { abraded }\end{array}$ & Gastric infusion \\
\hline Normal & Yes & No & No \\
\hline Model & Yes & Yes & No \\
\hline Control & Yes & Yes & $\begin{array}{c}10 \mathrm{~mL} / \mathrm{kg} \text { normal } \\
\text { saline }\end{array}$ \\
\hline Low dose IFRD & Yes & Yes & $\begin{array}{c}10 \mathrm{~mL} / \mathrm{kg} \text { IFRD } \\
\text { (equivalent to } \\
7.55 \mathrm{~g} \text { of herbs/kg) }\end{array}$ \\
\hline High dose IFRD & Yes & Yes & $\begin{array}{c}10 \mathrm{~mL} / \mathrm{kg} \text { IFRD } \\
\text { (equivalent to } 15.1 \mathrm{~g} \\
\text { of herbs/kg) }\end{array}$ \\
\hline
\end{tabular}

The IFRD was administered to the rats twice a day via gastric infusion from postoperative day 0 to day 7 with $10 \mathrm{~mL} / \mathrm{kg}$ /day solution. In the high dose IFRD group, the dosage is equivalent to $15.1 \mathrm{~g}$ of crude herbs/ $\mathrm{kg} / \mathrm{day}$, which was calculated from the body surface area and is generally used clinically for humans [11]. In the low dose IFRD group, the dosage per rat is $7.55 \mathrm{~g}$ of crude herb/kg/day, which is equal to approximately half of the human dose. For rats in the control group, normal saline was administered at the same volume for rats in the IFRD groups. Each rat was monitored for changes in body weight. Every step of the operation and the postoperative interventions for each group are shown in Table 2.

2.3. Assessment of Adhesion Grade. Seven days after the operation, all the rats were anesthetized, and the abdominal cavities were opened with U-like incisions. The intraabdominal adhesion evaluation was performed by a single researcher blinded to the treatment data according to the standard adhesion grades by Nair et al. [12]. These standard grades are listed in Table 3.

\subsection{Haematoxylin and Eosin (HE) Staining and Microscopic} Histological Grading. The injured peritoneum and adhesion tissues were excised after assessment of the adhesion grade. Specimen fixation and section preparation were carried out 
TABLE 3: The numerical scoring of adhesion described by Nair et al.

\begin{tabular}{ll}
\hline Grade & Criteria \\
\hline 0 & $\begin{array}{l}\text { No adhesion band is present } \\
1\end{array}$ \\
& $\begin{array}{l}\text { A single adhesion band forms between the viscera or } \\
\text { between a viscus and the abdominal wall } \\
\text { Two bands form between the viscera or between the } \\
\text { viscera and abdominal wall } \\
\text { More than two bands form between the viscera or } \\
\text { between the viscera and abdominal wall, or the } \\
\text { whole intestine forms a mass without adhering to the } \\
\text { abdominal wall } \\
\text { The viscera have directly adhered to the abdominal } \\
\text { wall, irrespective of the number of bands }\end{array}$ \\
&
\end{tabular}

and then HE staining was performed. The tissues were evaluated under a microscope as previously reported [13]. The evaluation standards were as follows: degree of inflammation (grade 0: absent or normal in number; grade 1: slight increase; grade 2: moderate infiltration; and grade 3: massive infiltration), fibrosis (grade 0: none; grade 1: slight; grade 2: moderate; and grade 3: dense), and neovascularization (grade 0: none; grade 1: 1-2 vessels; grade 2: 3-9 vessels; and grade 3: 10 or more vessels).

2.5. Immunohistochemistry. The samples were fixed with $4 \%$ paraformaldehyde and then embedded in paraffin. After cutting the paraffin-embedded samples into sections, immunohistochemical staining was performed. The expression level of $\alpha$-SMA (sc-53015, 1:800 dilution; Santa Cruz Biotechnology, Dallas, TX, USA) was detected. An image signal acquisition and analysis system (Leica, Wetzlar, Germany) was used for image acquisition.

2.6. Enzyme-Linked Immunosorbent Assay (ELISA). Seven days after surgery, blood samples from the rats were obtained. After centrifugation at $3,000 \mathrm{rpm}$ for $30 \mathrm{~min}$, only the serum was retained and stored at $-20^{\circ} \mathrm{C}$. The serum levels of TNF$\alpha$ and IL- 6 were examined using the ELISA kit (R\&D, Minneapolis, MN, USA) according to the manufacturer's recommendations.

2.7. Statistical Analysis. All the data are expressed as the mean \pm standard error or the median. A one-way ANOVA was performed followed by the $L S D$ method for multiple comparisons to compare differences between the groups. A Kruskal-Wallis analysis was applied for assessing the adhesion grades. A Mann-Whitney $U$ analysis was used to compare intergroup differences. The statistical analysis was performed using SPSS software version 13.0. $P<0.05$ was considered to be statistically significant.

\section{Results}

3.1. The IFRD Reduced the Macroscopic Grades of IntraAbdominal Adhesion in Rat Models. None of the rats died and all completed the experiment. The grades of adhesion showed significant differences between the different groups. There was almost no adhesion in the normal group (Figure 1(a)), whereas the rats in the model group (Figure 1(b)) and in the control group (Figure 1(c)) demonstrated patchy adhesion that could not be separated; the adhesion appeared at the injured areas of the peritoneum and caecum surface as well as the omentum. In contrast, the adhesions in the animals of the low dose IFRD group appeared to be loose and easy to separate (Figure $1(\mathrm{~d})$ ). The rats in the high dose IFRD group showed slight adhesions or even no adhesions. After grading the adhesions, the five groups showed significant differences $(P<0.05)$ (Table 4 and Figure $1(\mathrm{e}))$. There was no obvious difference in the adhesion grades between the model group and the control group, excluding the possibility of gastric infusion with an equal volume of saline for adhesion prevention. Compared to the control group, we found that the IFRD, especially the high dose of IFRD, significantly reduced the grades of adhesion. Thus, the results showed that the IFRD was able to prevent intra-abdominal adhesion in rats.

3.2. The IFRD Inhibited Inflammation, Fibrosis, and Neovascularization in Rat Models. By assessing the HE staining of the adhesion and peritoneum wound tissue slices, the grades of inflammation, fibrosis, and neovascularization were observed (Figure 2). We found that compared with the normal group the grades of inflammation, fibrosis, and neovascularization in the model and control groups were improved. However, compared to the control group, the high dose IFRD group distinctly presented less inflammation, fibrosis, and neovascularization (Figure 3). There is a trend to reduce inflammation, fibrosis, or neovascularization in the low dose IFRD group compared with the control group. Therefore, the data indicate that the IFRD inhibited inflammation, fibrosis, and neovascularization in the progression of adhesion induced by abrasion.

\subsection{The IFRD Inhibited $\alpha-S M A$, an Activated Fibroblast} Marker, in Adhesion Tissues in the Rat Model. To further study the degree of fibrosis of adhesion tissues in the rat model, immunohistochemical staining of $\alpha$-SMA, an activated fibroblast marker, was performed. In the normal group, no positive staining in the intact peritoneum was observed. In the model and control groups, there were a large number of fusiform fibroblasts with positive brown staining in the thick adhesive tissue. However, in the high dose IFRD group, the expression of $\alpha$-SMA in the injured peritoneum and/or adhesion tissues was remarkably decreased compared to the model and control groups (Figure 4).

3.4. The IFRD Suppressed the Blood Levels of TGF- $\beta$ and IL6 in the Rat Model. We used ELISA to analyse the levels of TGF- $\beta 1$ and IL- 6 in the serum. The results indicate that the serum levels of TGF- $\beta$ (Figure 5(a)) and IL-6 (Figure 5(b)) were notably higher in the model and control groups than that in the normal group, suggesting that operative injuryinduced adhesion formation was accompanied by significant inflammatory response. A high dose of IFRD can markedly inhibit the increase of TGF- $\beta$ and IL-6 $(P<0.05)$. 


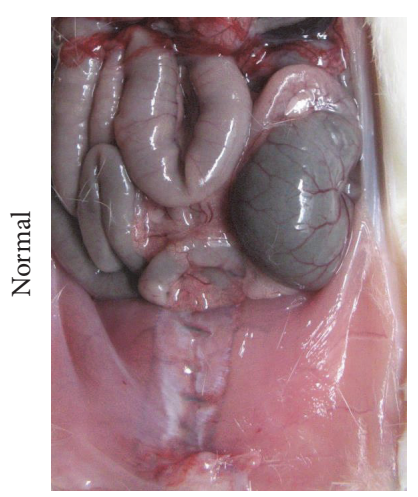

(a)

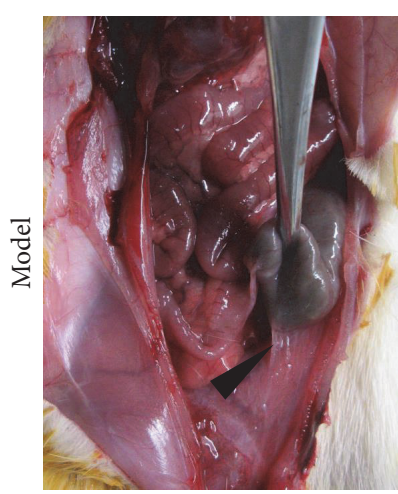

(b)

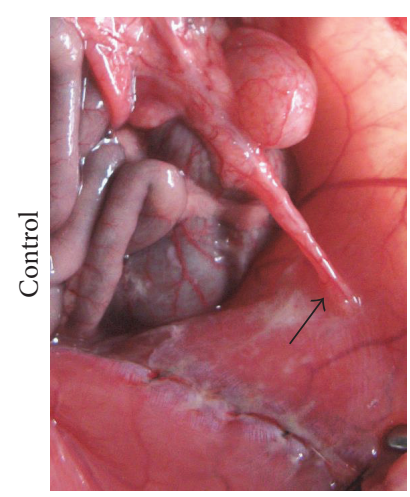

(c)

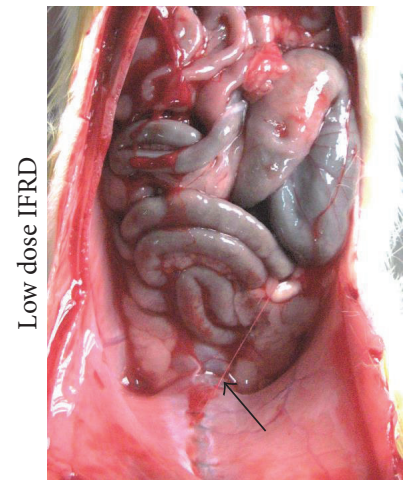

(d)

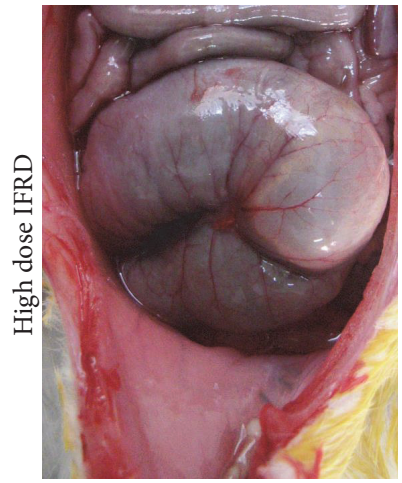

(e)

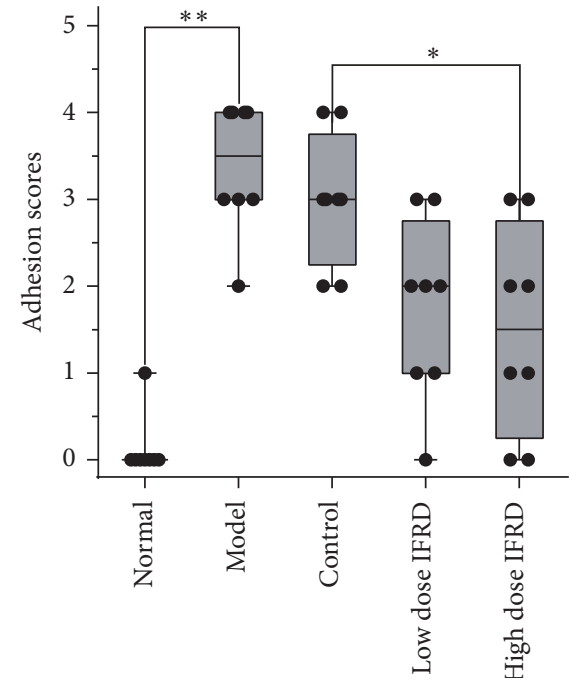

(f)

FIGURE 1: The intestine function recovery decoction (IFRD) prevented intra-abdominal adhesion formation in rats. (a) Normal group animals had intra-abdominal adhesions. (b) Model group animals developed a large number of extensive, thick adhesions, which were difficult to separate (black triangle). (c) Intra-abdominal adhesions occurred only slightly less in control group animals (black arrow). (d) The low dose IFRD group exhibited fewer intra-abdominal adhesions (black arrow) than the control group. (e) In the high dose IFRD group, some animals had no intra-abdominal adhesions. (f) Adhesion scores for the acroscopic classification $(n=8)$. The IFRD groups had the lowest scores of adhesions $\left({ }^{*} P<0.05 ;{ }^{* *} P<0.01\right)$.

TABLE 4: Effect of IFRD on rat abdominal adhesions after operation.

\begin{tabular}{lllllllcc}
\hline \multirow{2}{*}{ Group } & $n$ & \multicolumn{4}{c}{ Adhesions degree classification } & \multicolumn{2}{c}{ Mean \pm SD } & Median adhesion score \\
& & 0 & 1 & 2 & 3 & 4 & 0 \\
\hline Normal & 8 & 7 & 1 & 0 & 0 & 0 & $0.125 \pm 0.331$ & 3.5 \\
Model & 8 & 0 & 0 & 1 & 3 & 4 & $3.375 \pm 0.696$ & 3 \\
Control & 8 & 0 & 0 & 2 & 4 & 2 & $3 \pm 0.707$ & 2 \\
Low dose IFRD & 8 & 1 & 2 & 3 & 2 & 0 & $1.75 \pm 0.968$ & 1.5 \\
High dose IFRD & 8 & 2 & 2 & 2 & 2 & 0 & $1.5 \pm 1.118$ & $<0.01$ \\
$P^{\#}$ & & & & & & & & \\
\hline
\end{tabular}

${ }^{\#}$ Kruskal-Wallis analysis.

\section{Discussion}

Intra-abdominal adhesions are a common complication after abdominal and pelvic surgery [5]. Our study showed that the TCM IFRD could effectively prevent postoperative intra-abdominal adhesions in a rat model, likely resulting from the inhibition of inflammation, fibrosis, and neovascularization during adhesion formation.

An abdominal adhesion forms between two wound surfaces of the peritoneum. The formation of an adhesion depends on whether the deposited fibrous tissue is absorbed or undergoes organization [14]. The factors resulting in 


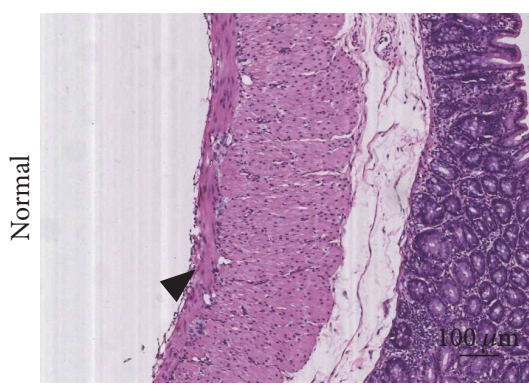

(a)

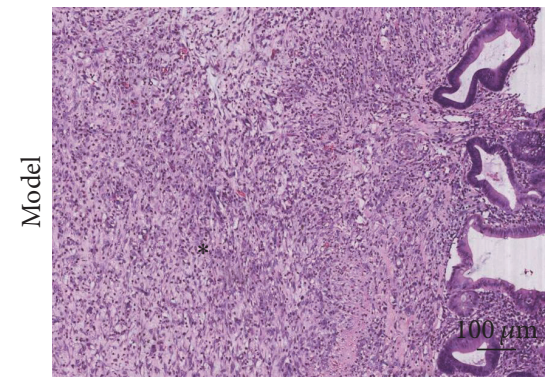

(b)

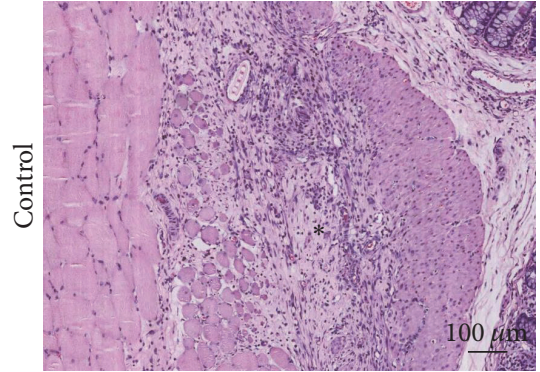

(c)

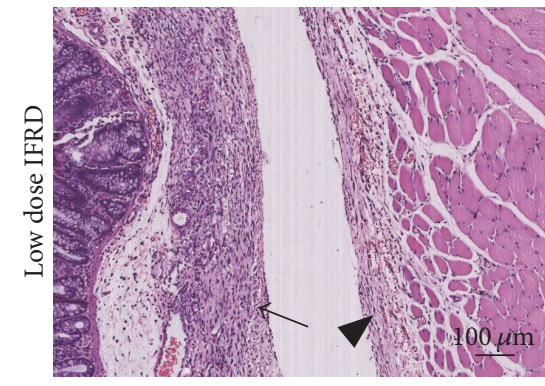

(d)

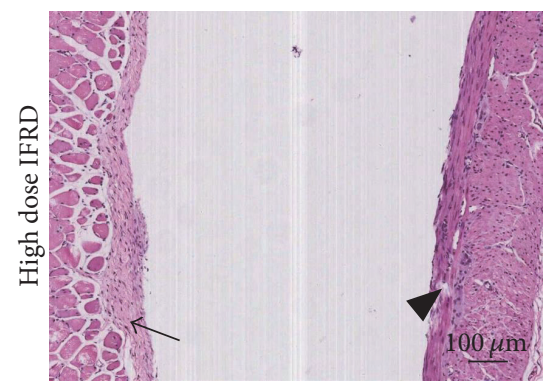

(e)

FiguRE 2: Representative images of HE staining in each group (100x). (a) Normal group; (b) model group; (c) control group; (d) low dose IFRD group; and (e) high dose IFRD group (* indicates the area of adhesion tissue; black triangle indicates visceral peritoneum; black arrow indicates parietal peritoneum).

adhesion formation include peritoneal mechanical trauma and peritoneal ischaemia caused by operation, traction, or residue from foreign matter, such as a suture. All these factors cause damage to the peritoneum and an inflammatory response. The cytokines released by inflammatory cells and oxidative stress are considered triggers and important initial events. The inflammatory reaction caused by peritoneal damage will produce fibrous exudation and deposition; meanwhile, the dissolving capacity of wound tissue will decrease. Ultimately, the deposition of extracellular matrix results in the formation of an adhesion $[15,16]$ Thus, the formation of an adhesion is a complex process caused by different cells, inflammatory mediators, and cytokines.

With the mechanism of adhesion formation elucidated, many preventative methods have emerged. Several barrier materials have been used clinically, including hyaluronic acid, carboxymethyl chitin, and oxidized regenerated cellulose, to prevent adhesion by separating the wounded tissues and promoting the repair of mesothelium cells [17-19]. Furthermore, researchers have attempted to prevent postoperative adhesion by inhibiting the inflammatory response [20], regulating fibrinolysis [21], and using antiangiogenesis [22] and antifibrosis [23] methods. However, verification of the value, effectiveness, and safety of these applications requires clinical trials and evidence-based medicine.

The IFRD consists of nine different herbs that have complex chemical components. According to the TCM theory $[24,25]$, the IFRD plays an important role in the special therapeutic method of "removing stasis by purgation and promoting blood circulation to remove blood stasis." The IFRD works effectively in treating severe abnormal infection and bowel motility dysfunction. In essence, the IFRD promotes tissue repair, decreases the inflammatory reaction and exudation, improves circulation in the intestine, and eventually improves intestinal functional recovery. Our study shows that the grades of intra-abdominal adhesion were reduced and the nonadhesion percentage was elevated in rats given the IFRD. Consequently, the IFRD may have an advantage in potentially preventing intra-abdominal adhesion.

Studies have demonstrated that inhibiting the inflammatory reaction can prevent intra-abdominal adhesion in animals $[26,27]$. Thus, it may be effective in preventing adhesion by inhibiting the inflammatory reaction and cytokines induction caused by injury. In the IFRD, some constituents including Codonopsis Radix (Dangshen) [28], Atractylodes Rhizoma (Baizhu) [29], and Paeoniae Rubra Radix (Chishao) [30] were reported to have anti-inflammatory and antioxidative effects. Jia and $\mathrm{He}$ [31] indicated that paeoniflorin, a chemical constituent of Paeoniae Rubra Radix, ameliorated disease in rat models of rheumatoid arthritis by suppressing oxidative stress and inflammation and reducing COX-2 protein expression. Wei et al. [32] and other researchers $[20,33]$ have provided evidence that hypoxia-induced COX2 expression in peritoneal fibroblasts is involved in the formation of intra-abdominal adhesions. In the current study, the grade of inflammation in the IFRD group was lower than that in the control group, which demonstrates that the IFRD possesses potent anti-inflammatory properties.

Cytokines such as IL- 6 and TGF- $\beta$ are of vital importance during the formation of adhesions. IL- 6 is a key cytokine for regulating the proliferation of epithelial cells and promoting the deposition of inflammatory cells and fibrosis at damage 


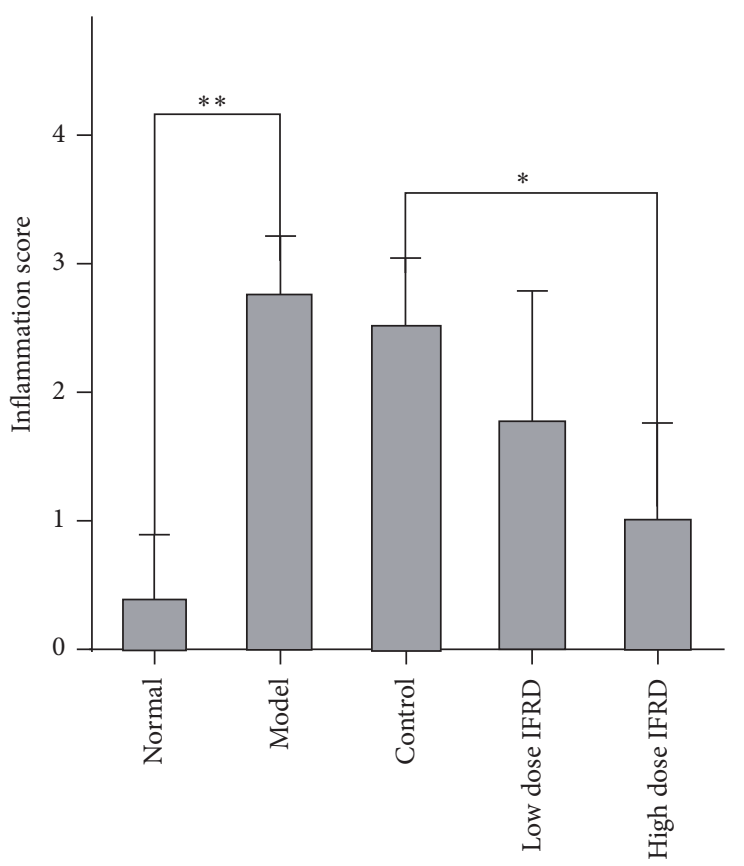

(a)

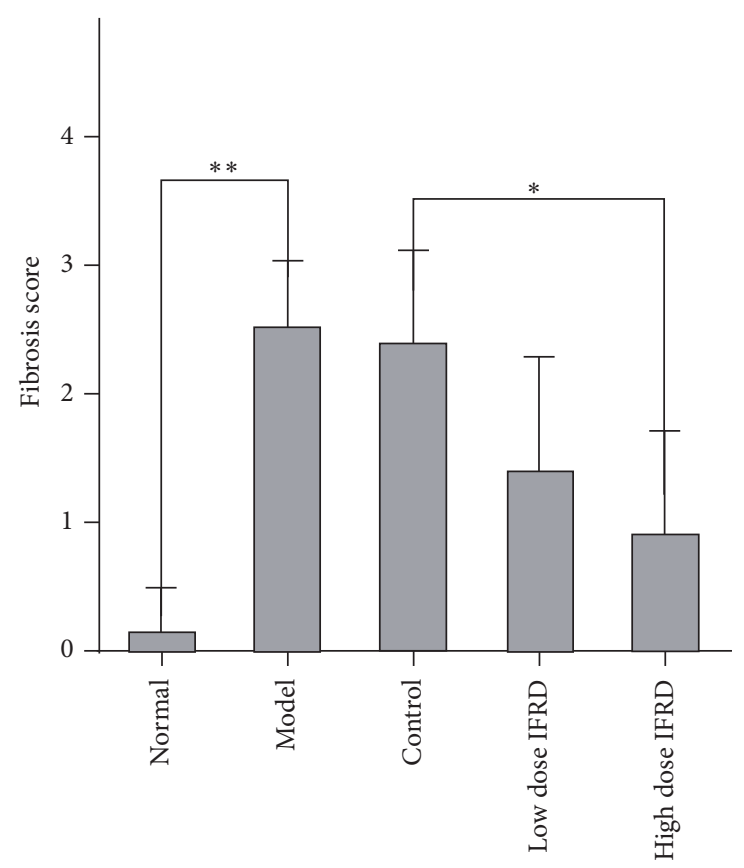

(b)

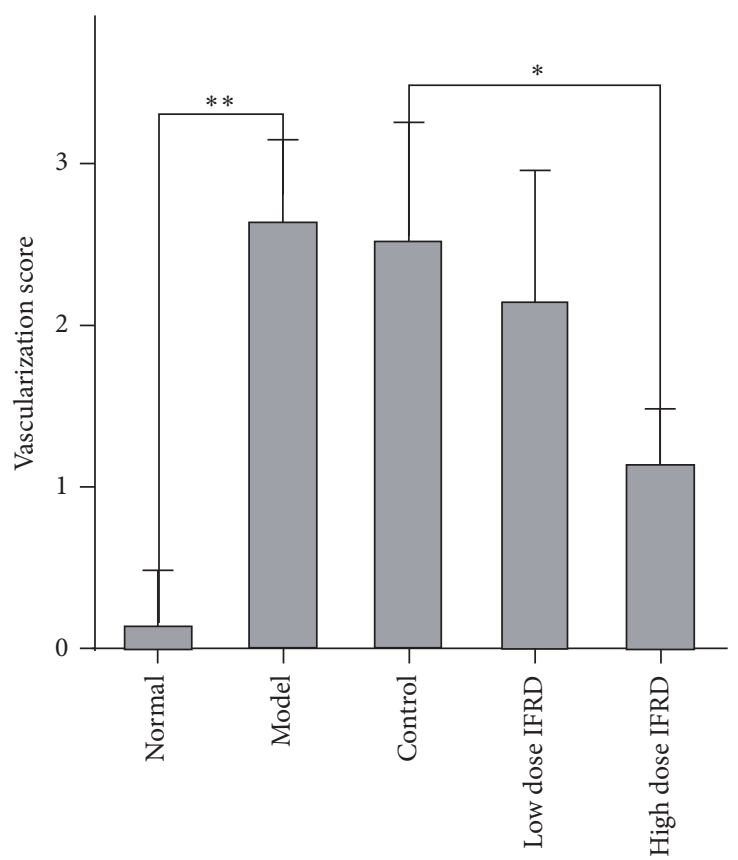

(c)

FIGURE 3: Inflammation, fibrosis, and neovascularization scores for each group $\left({ }^{*} P<0.05\right.$; $\left.{ }^{* *} P<0.01\right)$. (a) Inflammation scores, (b) fibrosis scores, and (c) neovascularization scores are shown.

sites [34]. TGF- $\beta$ promotes peritoneal mesothelial cells to increase the synthesis of plasminogen activator inhibitor1 by regulating the activity of various cytokines [35] and accelerates the migration and proliferation of adhesion fibroblasts [23]. The expression levels of IL-6 and TGF- $\beta$ positively regulate the formation of adhesions. Our present study suggests that the preventative effects of the IFRD on intra-abdominal adhesion formation are involved in inhibition of inflammatory cytokines IL- 6 and TGF- $\beta$ release.

Because the overexpression of cytokines induced by inflammation correlates with hyperplasia of fibrous connective tissue, the IFRD may work by inhibiting the expression of cytokines and decreasing hyperplasia of fibrous connective tissue. Fibroblast cells are crucial for ECM deposition; once 


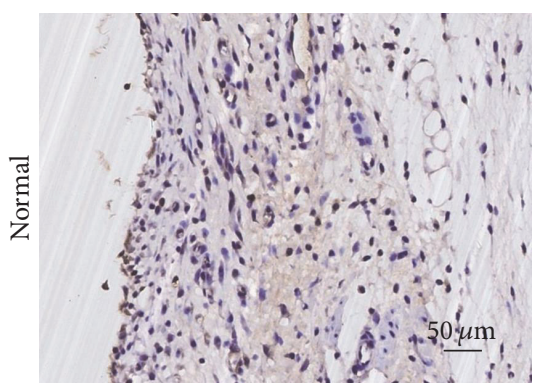

(a)

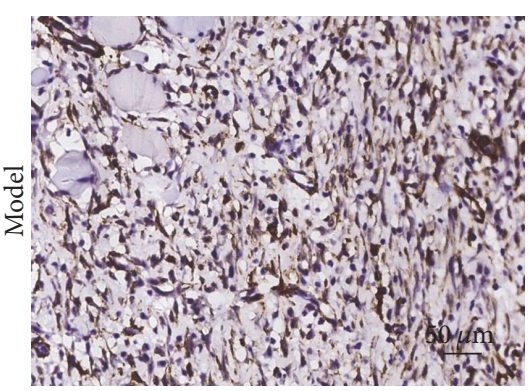

(b)

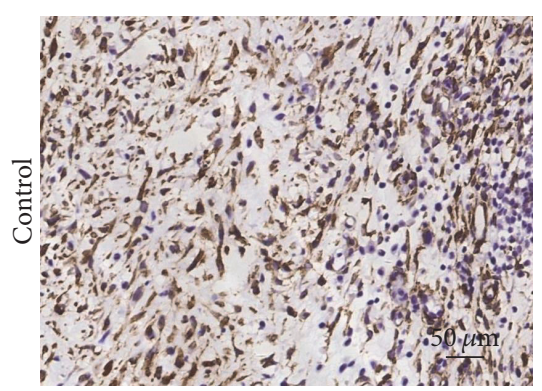

(c)

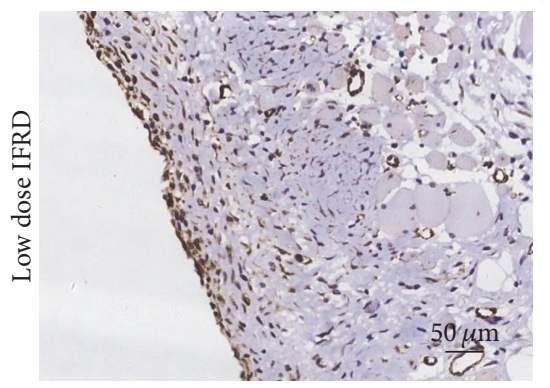

(d)

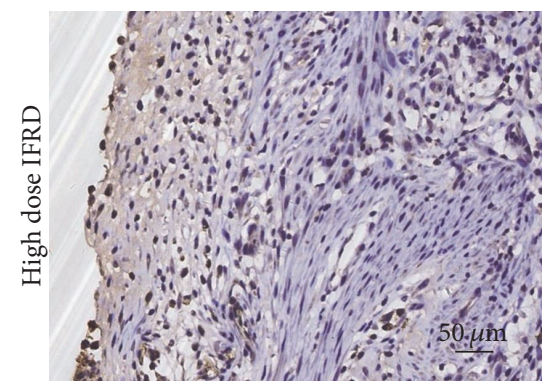

(e)

FIGURE 4: Immunohistochemical analysis of $\alpha$-SMA in intra-abdominal adhesion tissues from all groups (200x). (a) Normal group; (b) model group; (c) control group; (d) low dose IFRD group; and (e) high dose IFRD group.

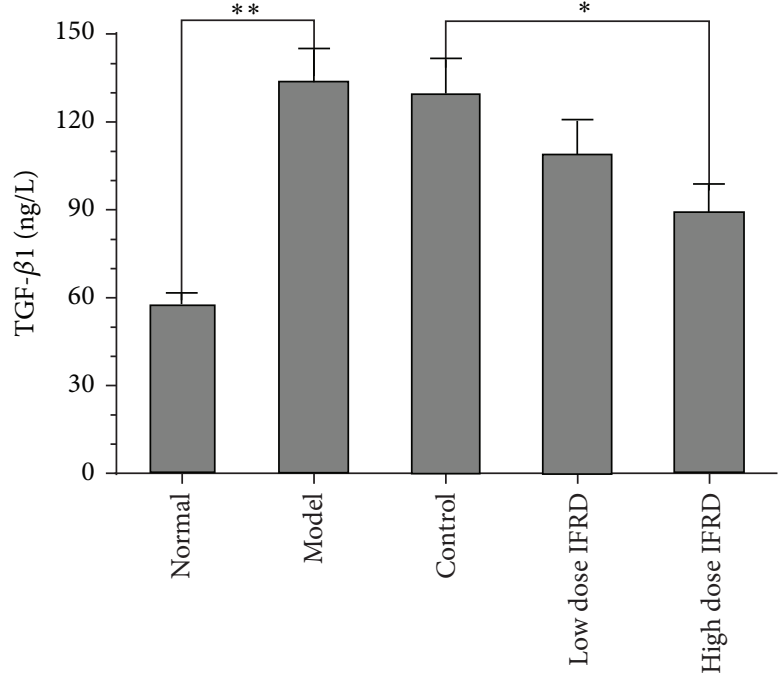

(a)

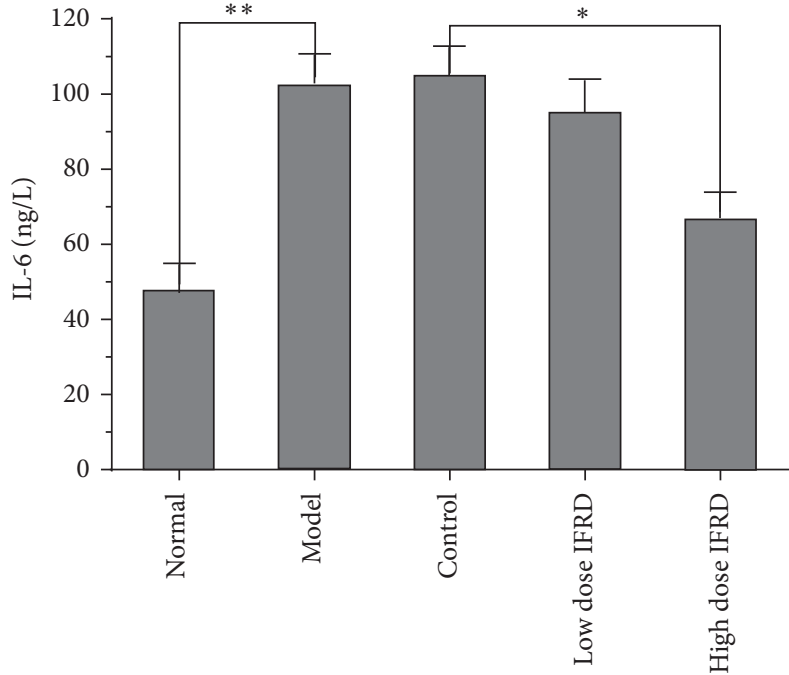

(b)

Figure 5: Effects of the IFRD on the serum levels of TGF- $\beta 1$ (a) and IL-6 (b) $\left({ }^{*} P<0.05 ;{ }^{* *} P<0.01\right)$.

activated, they differentiate into myofibroblasts that express $\alpha$-SMA [30]. A hallmark of myofibroblast activation, $\alpha$-SMA, is typically used to assess the level of fibrosis [36], and positive staining of $\alpha$-SMA reveals the development of fibrosis. In our results, the observation of decreased fibrosis grades and $\alpha$ SMA expression in the IFRD group demonstrated that the IFRD notably inhibited fibrosis and activated fibroblasts in adhesion formation.

Operative injury damages the balance between the production and degradation of fibrous protein while inducing the deposition of ECM, which forms the foundation of the adhesion. Persicae Semen (Taoren) in the IFRD is a representative herb for invigorating blood circulation and eliminating stasis; after thousands of years of clinical application and observation in China, its therapeutic effects are indeed certain, and its pharmacoactivity is defined [37]. Persicae Semen (Taoren) significantly decreased fibrinogen content, prolonged thrombin time and thromboplastin time, and increased prothrombin time in an animal model [38]. Therefore, the mechanism of action of the IFRD for adhesion 
prevention may be related to activating the fibrinolytic system and decreasing the deposition of fibrous protein.

An intra-abdominal adhesion may have restricted motility in the slow postoperative recovery of intestinal function caused by injury from the abdominal operation and anesthetization [39]. Inhibition of postoperative intestinal motility by inhibitors of gastrointestinal motility can result in increased numbers of adhesions. Therefore, the possibility of preventing postoperative adhesions by promoting gastrointestinal transit has been suggested [40]. Bove and Chapelle [41] have shown that visceral massage immediately following surgery interfered with postoperative adhesion formation by promoting normal peristaltic movements in a rat model. Some components of the IFRD have important effects on improving the peristalsis and movement of gastrointestinal smooth muscle. Rhei Radix et Rhizoma (Daihuang), Aucklandiae Radix (Muxiang), and Cannabis Fructus (Huomaren) have been used extensively for treating gastrointestinal motor dysfunction [42, 43]. Emodin, an anthraquinone derivative of Rhei Radix et Rhizoma (Daihuang), has also been reported to possess an anti-inflammatory effect [44]. Furthermore, Aurantii Fructus (Zhiqiao) and Magnoliae Officinalis Cortex (Houpu) used together can synergistically increase gut motor function by involving muscarinic receptors and secondarily alpha-receptors [45]. Therefore, the IFRD is applied to promote the recovery of dynamic intestinal function and shorten the contact time with impaired peritoneum lining, thus preventing intra-abdominal adhesion in rats.

The present study has some limitations that should be noted and further explored to elucidate precise mechanisms. Further studies are required. In a future study, we will try to elucidate these mechanisms and identify which constituents or pure compounds serve as active ingredients for adhesion prevention in the IFRD.

\section{Conclusion}

Our study found that the IFRD effectively prevented the formation of adhesions by decreasing inflammatory infiltration of the injured peritoneum and by reducing collagen deposition and fibrosis (Figure 6). This study demonstrated the wide-ranging potential value of the IFRD, a traditional Chinese medicine, in preventing postoperative adhesions, which could improve patient quality of life.

\section{Competing Interests}

The authors declare that there is no conflict of interests related to this work.

\section{Acknowledgments}

This study was supported by the National Natural Science Foundation of China (no. 81572734), the Scientific and Technological Development Research Project Foundation by Shaanxi Province (2016SF-121), and the Fundamental Research Funds for the Central Universities in Xi'an Jiaotong University (no. 2013jdhz33).

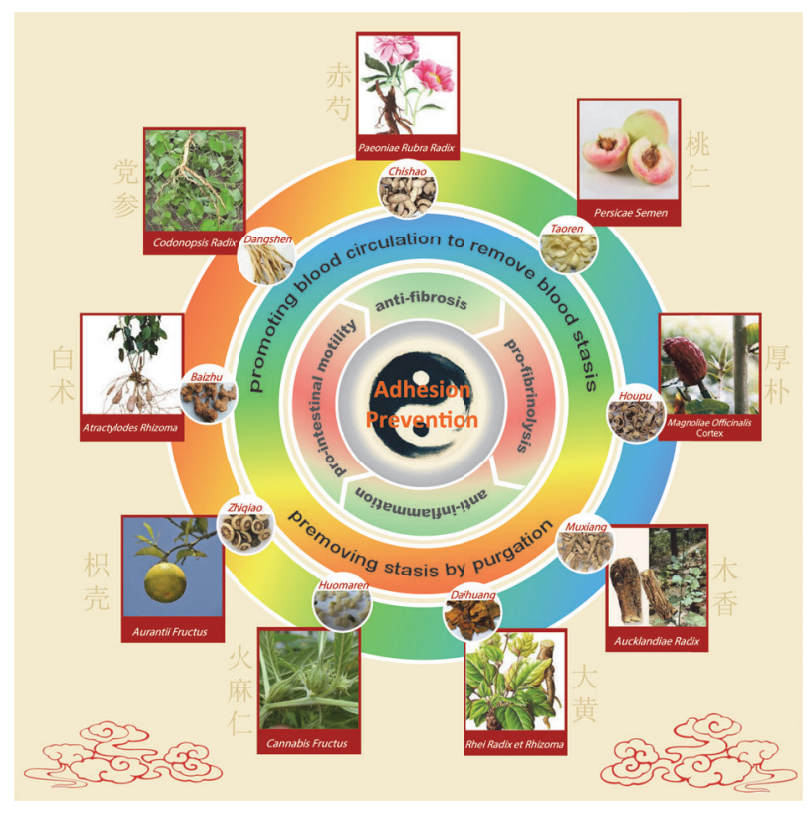

FIGURE 6: Preventative effects of the intestine function recovery decoction, a traditional Chinese medicine, on postoperative intraabdominal adhesion formation in a rat model. The outermost circle illustrates the nine traditional Chinese medicinal (TCM) herbs, which are components of the intestine function recovery decoction (IFRD). The second circle shows that the IFRD possesses two types of TCM pharmacology, namely, "removing stasis by purgation" and "promoting blood circulation to remove blood stasis." The third circle illustrates four types of underlying pharmacological action of the IFRD for preventing adhesive formation, including anti-inflammation, antifibrosis, profibrinolysis, and prointestinal motility. The kernel shows that the IFRD can effectively prevent adhesive formation independent of complementary TCM.

\section{References}

[1] R. T. Beyene, S. L. Kavalukas, and A. Barbul, "Intra-abdominal adhesions: anatomy, physiology, pathophysiology, and treatment," Current Problems in Surgery, vol. 52, no. 7, pp. 271-319, 2015.

[2] A. Barbul, "In brief. Abdominal adhesions," Current Problems in Surgery, vol. 52, no. 7, pp. 266-269, 2015.

[3] W. Arung, M. Meurisse, and O. Detry, "Pathophysiology and prevention of postoperative peritoneal adhesions," World Journal of Gastroenterology, vol. 17, no. 41, pp. 4545-4553, 2011.

[4] K. Okabayashi, H. Ashrafian, E. Zacharakis et al., "Adhesions after abdominal surgery: a systematic review of the incidence, distribution and severity," Surgery Today, vol. 44, no. 3, pp. 405420, 2014.

[5] J.-J. Duron, N. J.-D. Silva, S. T. Du Montcel et al., "Adhesive postoperative small bowel obstruction: incidence and risk factors of recurrence after surgical treatment: a multicenter prospective study," Annals of Surgery, vol. 244, no. 5, pp. 750757, 2006.

[6] B.-T. S. Fevang, J. Fevang, S. A. Lie, O. Søreide, K. Svanes, and A. Viste, "Long-term prognosis after operation for adhesive small bowel obstruction," Annals of Surgery, vol. 240, no. 2, pp. 193201, 2004. 
[7] R. P. G. ten Broek, Y. Issa, E. J. P. van Santbrink et al., "Burden of adhesions in abdominal and pelvic surgery: systematic review and met-analysis," British Medical Journal, vol. 347, no. 7929, Article ID f5588, 2013.

[8] J. M. Becker and A. F. Stucchi, "Intra-abdominal adhesion prevention: are we getting any closer?" Annals of Surgery, vol. 240, no. 2, pp. 202-204, 2004.

[9] W. B. Robb and C. Mariette, "Strategies in the prevention of the formation of postoperative adhesions in digestive surgery: a systematic review of the literature," Diseases of the Colon and Rectum, vol. 57, no. 10, pp. 1228-1240, 2014.

[10] C. C. Peyton, T. Keys, S. Tomblyn et al., "Halofuginone infused keratin hydrogel attenuates adhesions in a rodent cecal abrasion model," Journal of Surgical Research, vol. 178, no. 2, pp. 545-552, 2012.

[11] S. Reagan-Shaw, M. Nihal, and N. Ahmad, "Dose translation from animal to human studies revisited," FASEB Journal, vol. 22, no. 3, pp. 659-661, 2008.

[12] S. K. Nair, I. K. Bhat, and A. L. Aurora, "Role of proteolytic enzyme in the prevention of postoperative intraperitoneal adhesions," Archives of Surgery, vol. 108, no. 6, pp. 849-853, 1974.

[13] A. Sahbaz, O. Aynioglu, H. Isik, B. D. Gun, O. Cengil, and O. Erol, "Pycnogenol prevents peritoneal adhesions," Archives of Gynecology and Obstetrics, vol. 292, no. 6, pp. 1279-1284, 2015.

[14] N. Chegini, "Peritoneal molecular environment, adhesion formation and clinical implication," Frontiers in Bioscience, vol. 7, pp. e91-e115, 2002.

[15] G. S. DiZerega and J. D. Campeau, "Peritoneal repair and postsurgical adhesion formation," Human Reproduction Update, vol. 7, no. 6, pp. 547-555, 2001.

[16] Y. C. Cheong, S. M. Laird, T. C. Li, J. B. Shelton, W. L. Ledger, and I. D. Cooke, "Peritoneal healing and adhesion formation/reformation," Human Reproduction Update, vol. 7, no. 6, pp. 556-566, 2001.

[17] U. S. Ha, J. S. Koh, K. J. Cho et al., "Hyaluronic acidcarboxymethylcellulose reduced postoperative bowel adhesions following laparoscopic urologic pelvic surgery: a prospective, randomized, controlled, single-blind study," BMC Urology, vol. 16, no. 1, article 28, 2016.

[18] X. Wu, J. Hu, D. Fan et al., "Safety and efficacy of sodium hyaluronate gel and chitosan in preventing postoperative peristomal adhesions after defunctioning enterostomy: a prospective randomized controlled trials," Medicine (United States), vol. 94, no. 51, article no. e2354, 2015.

[19] G. Wei, C. Zhou, G. Wang, L. Fan, K. Wang, and X. Li, "Keratinocyte growth factor combined with a sodium hyaluronate gel inhibits postoperative intra-abdominal adhesions," International Journal of Molecular Sciences, vol. 17, no. 10, 2016.

[20] Y. I. Kim, "Comparative study for preventive effects of intraabdominal adhesion using cyclo-oxygenase- 2 enzyme (COX2) inhibitor, low molecular weight heparin (LMWH), and synthetic barrier," Yonsei Medical Journal, vol. 54, no. 6, pp. 1491-1497, 2013.

[21] P. Dinarvand, S. M. Hassanian, H. Weiler, and A. R. Rezaie, "Intraperitoneal administration of activated protein $\mathrm{C}$ prevents postsurgical adhesion band formation," Blood, vol. 125, no. 8, pp. 1339-1348, 2015.

[22] S. Kim, S. Lee, A. K. Greene et al., "Inhibition of intraabdominal adhesion formation with the angiogenesis inhibitor sunitinib," Journal of Surgical Research, vol. 149, no. 1, pp. 115119, 2008.
[23] L. Deng, Q. Li, G. Lin et al., "P-glycoprotein mediates postoperative peritoneal adhesion formation by enhancing phosphorylation of the chloride channel-3," Theranostics, vol. 6, no. 2, pp. 204-218, 2016.

[24] R. Teschke, A. Wolff, C. Frenzel, A. Eickhoff, and J. Schulze, "Herbal traditional Chinese medicine and its evidence base in gastrointestinal disorders," World Journal of Gastroenterology, vol. 21, no. 15, pp. 4466-4490, 2015.

[25] K.-J. Chen, "Blood stasis syndrome and its treatment with activating blood circulation to remove blood stasis therapy," Chinese Journal of Integrative Medicine, vol. 18, no. 12, pp. 891896, 2012.

[26] T. Dalgic, E. Oymaci, E. B. Bostanci et al., "Effects of carbon dioxide pneumoperitoneum on postoperative adhesion formation and oxidative stress in a rat cecal abrasion model," International Journal of Surgery, vol. 21, pp. 57-62, 2015.

[27] G. Wei, X. Chen, G. Wang, L. Fan, K. Wang, and X. Li, "Effect of resveratrol on the prevention of intra-abdominal adhesion formation in a rat model," Cellular Physiology and Biochemistry, vol. 39, no. 1, pp. 33-46, 2016.

[28] J. Y.-W. Chan, F.-C. Lam, P.-C. Leung, C.-T. Che, and K.-P. Fung, "Antihyperglycemic and antioxidative effects of a herbal formulation of Radix Astragali, Radix Codonopsis and Cortex Lycii in a mouse model of type 2 diabetes mellitus," Phytotherapy Research, vol. 23, no. 5, pp. 658-665, 2009.

[29] S. Ahmed, C. Zhan, Y. Yang et al., "The transcript profile of a traditional chinese medicine, Atractylodes lancea, revealing its sesquiterpenoid biosynthesis of the major active components," PLOS ONE, vol. 11, no. 3, Article ID e0151975, 2016.

[30] Y. Ji, Y. Dou, Q. Zhao et al., "Paeoniflorin suppresses TGF$\beta$ mediated epithelial-mesenchymal transition in pulmonary fibrosis through a Smad-dependent pathway," Acta Pharmacologica Sinica, vol. 37, no. 6, pp. 794-804, 2016.

[31] Z. Jia and J. He, "Paeoniflorin ameliorates rheumatoid arthritis in rat models through oxidative stress, inflammation and cyclooxygenase 2," Experimental and Therapeutic Medicine, vol. 11, no. 2, pp. 655-659, 2016.

[32] G. Wei, X. Chen, G. Wang et al., "Inhibition of cyclooxygenase2 prevents intra-abdominal adhesions by decreasing activity of peritoneal fibroblasts," Drug Design, Development and Therapy, vol. 9, pp. 3083-3098, 2015.

[33] A. K. Greene, I. P. J. Alwayn, V. Nose et al., "Prevention of intraabdominal adhesions using the antiangiogenic COX-2 inhibitor celecoxib," Annals of Surgery, vol. 242, no. 1, pp. 140-146, 2005.

[34] D. R. Ambler, N. M. Fletcher, M. P. Diamond, and G. M. Saed, "Effects of hypoxia on the expression of inflammatory markers IL-6 and TNF-a in human normal peritoneal and adhesion fibroblasts," Systems Biology in Reproductive Medicine, vol. 58, no. 6, pp. 324-329, 2012.

[35] G. Wang, K. Wu, W. Li et al., "Role of IL-17 and TGF- $\beta$ in peritoneal adhesion formation after surgical trauma," Wound Repair and Regeneration, vol. 22, no. 5, pp. 631-639, 2014.

[36] M. Kitamura, T. Nishino, Y. Obata et al., "The kampo medicine Daikenchuto inhibits peritoneal fibrosis in mice," Biological \& Pharmaceutical Bulletin, vol. 38, no. 2, pp. 193-200, 2015.

[37] S. Xi, L. Qian, H. Tong et al., "Toxicity and clinical reasonable application of Taoren (Semen Persicae) based on ancient and modern literature research," Journal of Traditional Chinese Medicine, vol. 33, no. 2, pp. 272-279, 2013.

[38] L. Liu, J.-A. Duan, Y. Tang et al., "Taoren-Honghua herb pair and its main components promoting blood circulation through 
influencing on hemorheology, plasma coagulation and platelet aggregation," Journal of Ethnopharmacology, vol. 139, no. 2, pp. 381-387, 2012.

[39] D. M. Scott-Coombes, M. N. Vipond, and J. N. Thompson, "Small bowel transit time in patients with intra-abdominal adhesions," The British Journal of Surgery, vol. 79, no. 10, p. 1076, 1992.

[40] F. Fu, Y. Hou, W. Jiang, R. Wang, and K. Liu, "Escin: inhibiting inflammation and promoting gastrointestinal transit to attenuate formation of postoperative adhesions," World Journal of Surgery, vol. 29, no. 12, pp. 1614-1622, 2005.

[41] G. M. Bove and S. L. Chapelle, "Visceral mobilization can lyse and prevent peritoneal adhesions in a rat model," Journal of Bodywork and Movement Therapies, vol. 16, no. 1, pp. 76-82, 2012.

[42] H. Guo, J. Zhang, W. Gao, Z. Qu, and C. Liu, "Gastrointestinal effect of methanol extract of Radix Aucklandiae and selected active substances on the transit activity of rat isolated intestinal strips," Pharmaceutical Biology, vol. 52, no. 9, pp. 1141-1149, 2014.

[43] R. L. Whiting and A. C. Ford, "Efficacy of traditional chinese medicine in functional constipation," The American Journal of Gastroenterology, vol. 106, no. 5, pp. 1003-1004, 2011.

[44] M. Xiao, T. Zhu, W. Zhang et al., "Emodin ameliorates LPSinduced acute lung injury, involving the inactivation of NF- $\kappa \mathrm{B}$ in mice," International Journal of Molecular Sciences, vol. 15, no. 11, pp. 19355-19368, 2014.

[45] X. Xiong, W. Peng, L. Chen et al., "Traditional Chinese medicine Zhiqiao-Houpu herb-pair induce bidirectional effects on gastric motility in rats," Journal of Ethnopharmacology, vol. 175, pp. 444-450, 2015. 


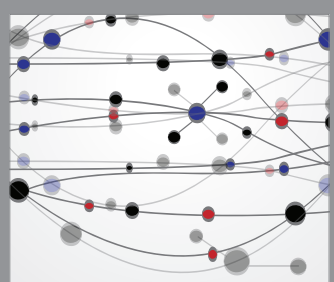

The Scientific World Journal
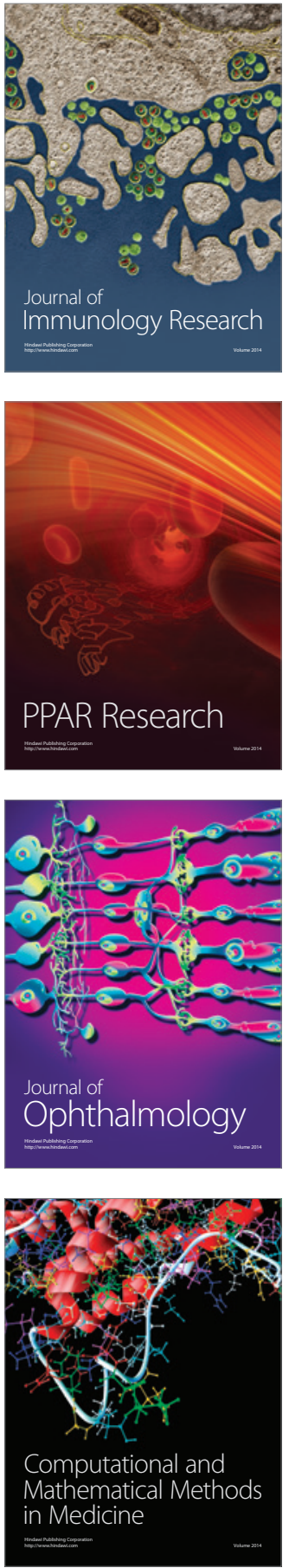

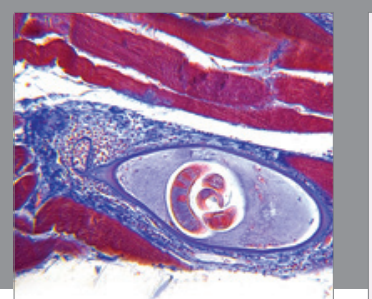

Gastroenterology Research and Practice

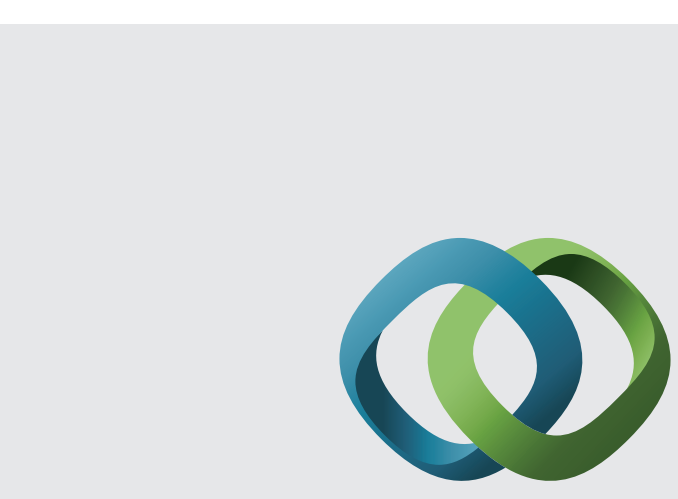

\section{Hindawi}

Submit your manuscripts at

http://www.hindawi.com
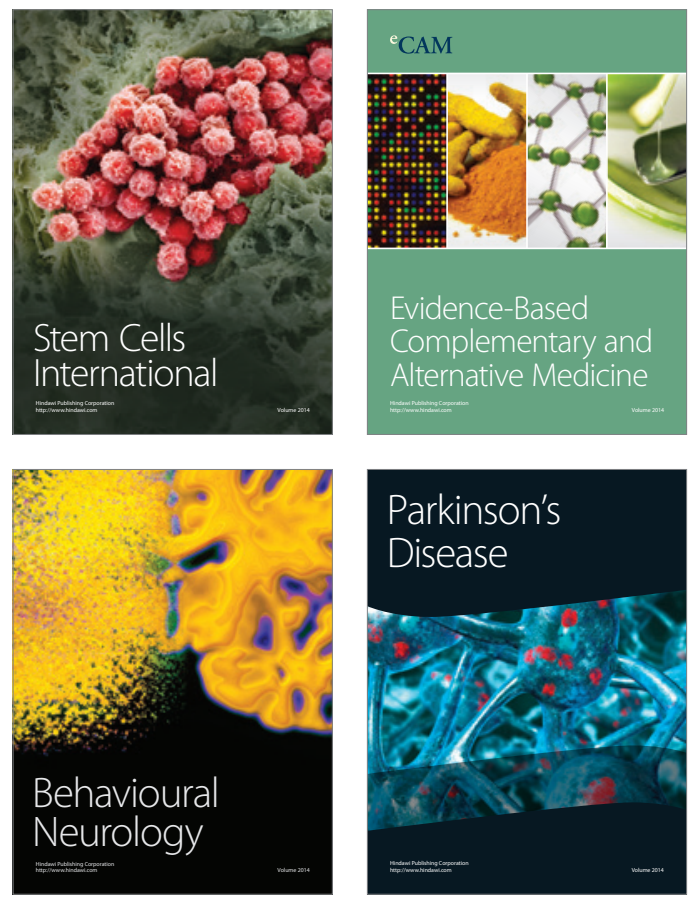
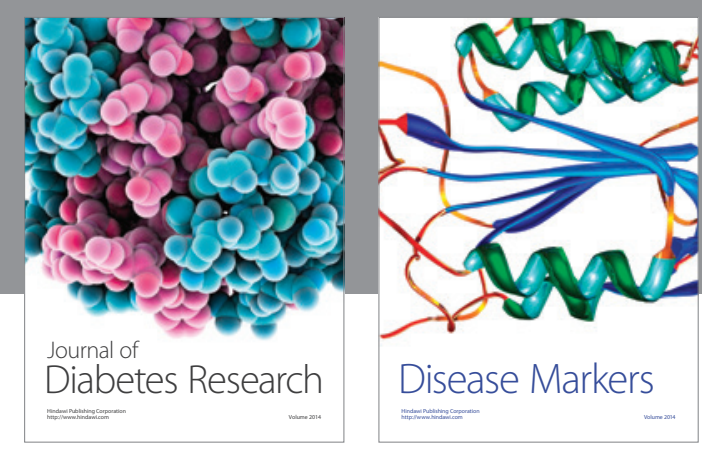

Disease Markers
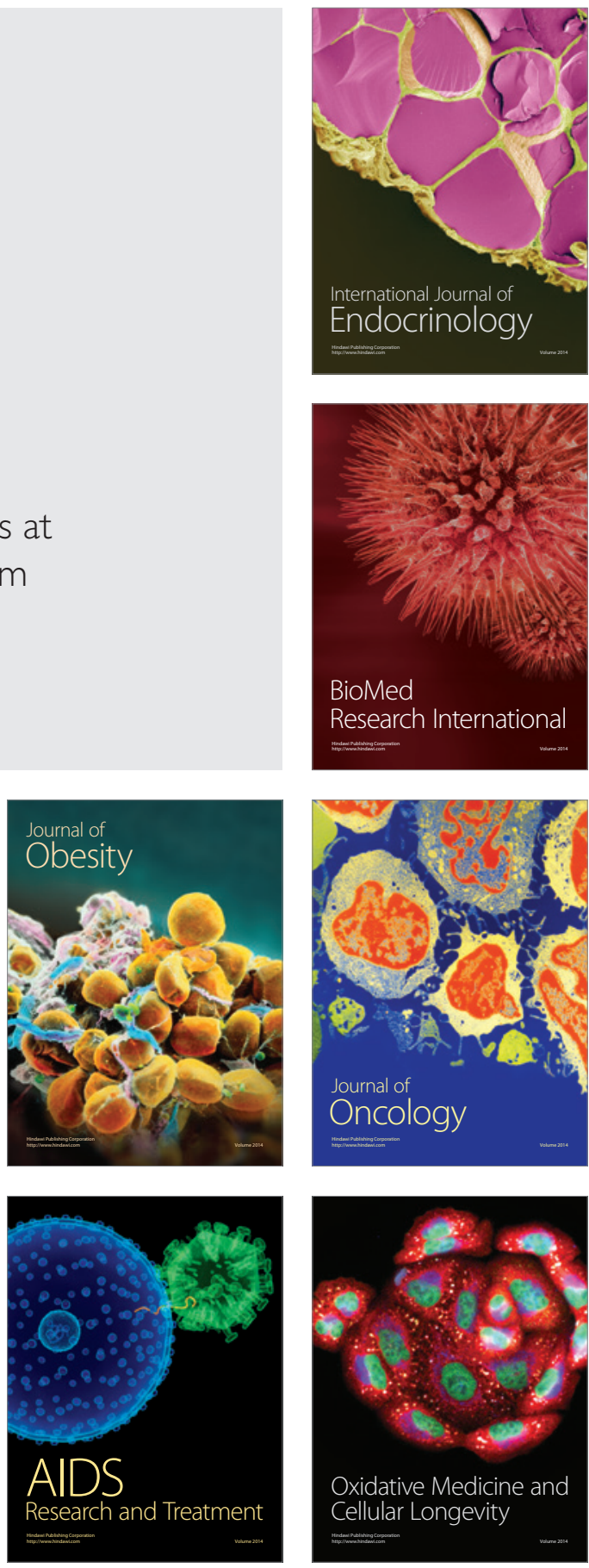